\title{
Thinking with/in/through Binaries and Boundaries: Sparking Necessary and Ongoing Conversations in Early Childhood Education
}

\author{
Iris Berger, Nancy van Groll, and Áurea Vericat Rocha, Guest Editors
}

SPARK: The Early Years is an early childhood education conference run by graduate students and held biennially at the University of British Columbia on the unceded traditional and ancestral Musqueam territory, colonially known as Vancouver, Canada. The acronym SPARK stands for supporting practice, research, and knowledge and speaks of the intent that brought this conference to life: namely, to support, gather, and connect the diverse and situated individual and collective efforts that sustain, nourish, and ignite new thinking and practice in early childhood education.

SPARK 2019 invited conference participants to explore and challenge the entrenched binaries and divides that still characterize conceptual and practical discussions in the ECE field. The conference theme "Surfacing Connections \& Challenging Divides: Research, Policy, and Practice in Early Childhood Education" inspired a sharing of innovative ways of thinking and acting that are surfaced when tensions are encountered and exposed. We conceived this publication to extend some of the boundary-crossing conversations that took place at the conference and also as a way to celebrate and disseminate the remarkable work that early childhood education students, educators, and scholars are doing in the field.

As we were nearing the publication of this issue, however, we could not ignore the unprecedented times we are all living in. Witnessing the surge of a global pandemic, the rightful revolt against racism and police brutality, and the imminent climate emergency provoked us to think about what new meanings emerge and what new relations can be made with the notions of borders and binaries. While it has been said that "we are all in this together," we are not one and the same (Braidotti, 2020). Ironically, as boundaries melted, borders have been shut down and we are obliged to be isolated and physically distanced from each other. A vast and growing majority continues to suffer much more than those of us who are privileged (see Braidotti, 2020, for a discussion of the inequalities the pandemic has surfaced). The isolation we are living in has revealed fractures in values and ideas, which, when in conflict with others', splinter further as a result of intolerance. News abounds with stories about hyperpolitical polarization and violent collisions of opposing political affiliations in response to local and global conditions. What has been sparked in 2020 has dramatically exposed extreme tensions and polarities, but also the intimate interconnections and entanglements that exist among all forms of life on earth.

These unprecedented times reinvigorate, we think, the importance of asking how early childhood education contributes to thinking and coming together differently. Responding to a question about our current condition, Emmanuel Vaughan-Lee (interviewed by Cheung, 2020) offers that the role of storytellers is to reveal chasms, and to share and dwell with experiences and perspectives, because stories unearth questions without rushing to provide answers. It is in this spirit that we share the "stories" in this special issue. All the authors featured in it answered a call to "lead us inside" their diverse and vibrant worlds and ideas. They engage with the complex intersections that exist in early childhood education and guide us through creative ways of thinking with/in/through binaries and boundaries.

Denise Hodgins, Narda Nelson, Sherri-Lynn Yazbeck, Xiaofeng Ke, and Rosalind Turcotte open this issue with "Living Speculative Pedagogies as Boundary-Crossing Dialogues." This paper beautifully illustrates how boundary crossing is enacted through a commitment to ongoing critically reflective dialogues among early childhood 
educators and pedagogists in an early childhood setting on the Songhees, Esquimalt, and W_SÁNEĆ traditional territories. While recounting a climate change inquiry project that explored thinking with trees, the authors show how ethics, collaboration, and curriculum can be thought of differently when committing to a boundary-crossing approach so that differences are made visible and therefore generate new possibilities.

Rachel Langford's article "Navigating Reconceptualist and Feminist Ethics of Care Scholarship to Find a Conceptual Space for Rethinking Children's Needs in Early Childhood Education" shares a fascinating conceptual journey that explores the boundaries between ECE reconceptualist and feminist ethics of care perspectives and invites us to reconsider the notion of children's needs. While reconceptualist scholars are critical of the tendency of developmental theory to focus on children's needs within a deficit approach, feminist ethics of care scholars view having needs and dependencies as ontologically central to being and living in a world where we are connected to one another via caring relationships. Articulating a third conceptual space for thinking about children's needs in ECE, Langford offers a compelling alternative to move beyond the developmental/reconceptualist binary.

Both Cayley Burton and Sarah Reddington problematize Western ideals of binary sex-gender performativity and how heteronormative boundaries work in early childhood contexts. In "Gender Disrupted During Storytime: Critical Literacy in Early Childhood Education," Cayley Burton suggests storytime as a context for a critical reading of gendered messages present in picture books featuring gender-nonconforming characters. Burton argues for gender disruption through critical literacy as necessary to achieving more inclusive ECE spaces. Sarah Reddington, taking a different approach to the study of gender performativity, focuses on early childhood educators' understandings of how children aged 4 to 5 perform gender during unstructured play. Among other findings, her research reveals that educators tend to view gender primarily through traditional gender stereotypes and often unknowingly construct heteronormative play spaces that then inform the ways in which children learn about gender.

In "Wrestling with 'Will to Truth' in Early Childhood Education: Cracking Spaces for Multiplicity and Complexity Through Poetry," Chenying Wang employs creative arts-based methodologies to challenge a conventional way to think, act, and feel as an early childhood educator. Through beautiful and evocative poetic practices, Wang illustrates a move beyond the assumed boundaries of a single image of an early childhood educator that welcomes the existence of multiple and complex subjectivities.

The special issue concludes with Lori Huston, Elder Brenda Mason, and Roxanne Loon's "Culturally Responsive Indigeneity of Relations," where they share the story of the Anishininiiwi Awaashishiiw Kihkinohamaakewi Niikaanihtamaakew Indigenous Early Childhood Education Leadership Program (IECELP) in Thunder Bay, Ontario. They discuss the wildfire circle as the Indigenous research method used in the IECELP as well as during their presentation at the SPARK 2019 conference. Their work foregrounds how dominant research paradigms have not addressed Indigenous peoples in meaningful and sustainable ways. In contrast, the IECELP values were grounded from the very beginning in Indigenous peoples' knowledge systems offered by Elder Brenda Mason. The authors propose alternative ways of sharing knowledge in Indigenous and culturally responsive ways.

Threading together this issue has afforded us the privilege of dwelling in the ways the authors illuminate how ECE is an active part of the world and its making, rather than the neutral and apolitical outsider it is often mistaken to be. We invite readers to witness the dynamic ways ECE is involved in challenging the status quo, rejecting exclusionary practices, and embodying micro politics of storytelling of the otherwise. By contextualizing this work in the emerging 2020 narratives, we are compelled to shift the question from boundaries to connections and ask: What new and unexplored interconnections might be generated during this time, and where might these connections take us? 


\section{Acknowledgments}

We would like to acknowledge the generous contributions the SPARK 2019 conference received from UBC's Early Childhood Education Program and the Centre for Early Childhood Education and Research, as well as from the Departments of Educational and Counselling Psychology, and Special Education; Educational Studies; Language and Literacy Education; Curriculum and Pedagogy; and Kinesiology. We also appreciate the contribution of UBC's Faculty of Education Dean's Office and the Office of Indigenous Education and that of the interdisciplinary Graduate Student Network. A special acknowledgment goes to other institutional funders that highlight the need and importance of interinstitutional collaboration in early childhood education: Simon Fraser University Childcare Society, Ryerson University, Early Childhood Educators of British Columbia, and the British Columbia Ministry of Children and Family Development. 


\section{References}

Braidotti, R. (2020). "We" are in this together, but we are not one and the same. Journal of Bioethical Inquiry. https://doi.org/10.1007/ $\underline{\text { s11673-020-10017-8 }}$

Cheung, J. (2020, August 10). Culture and health: A chat with Emmanuel Vaughan-Lee. Medium. https://medium.com/ideo-colab/ culture-and-health-a-chat-with-emmanuel-vaughan-lee-927541b08938 